\title{
Elevation Difference and Bouguer Anomaly Analysis Tool (EDBAAT) User's Guide
}

Open-File Report 2017-1036 



\section{Elevation Difference and Bouguer Anomaly Analysis Tool (EDBAAT) User's Guide}

By Aaron M. Smittle and Thomas G. Shoberg

Open-File Report 2017-1036 


\title{
U.S. Department of the Interior \\ RYAN K. ZINKE, Secretary
}

\section{U.S. Geological Survey William H. Werkheiser, Acting Director}

\author{
U.S. Geological Survey, Reston, Virginia: 2017
}

For more information on the USGS - the Federal source for science about the Earth, its natural and living resources, natural hazards, and the environment-visit https://www.usgs.gov or call 1-888-ASK-USGS.

For an overview of USGS information products, including maps, imagery, and publications, visit https://store.usgs.gov/.

Any use of trade, firm, or product names is for descriptive purposes only and does not imply endorsement by the U.S. Government.

Although this information product, for the most part, is in the public domain, it also may contain copyrighted materials as noted in the text. Permission to reproduce copyrighted items must be secured from the copyright owner.

Suggested citation:

Smittle, A.M., and Shoberg, T.G., 2017, Elevation Difference and Bouguer Anomaly Analysis Tool (EDBAAT) user's guide: U.S. Geological Survey Open File Report 2017-1036, 9 p., https://doi.org/10.3133/ofr20171036.

ISSN 2331-1258 (online) 


\section{Contents}

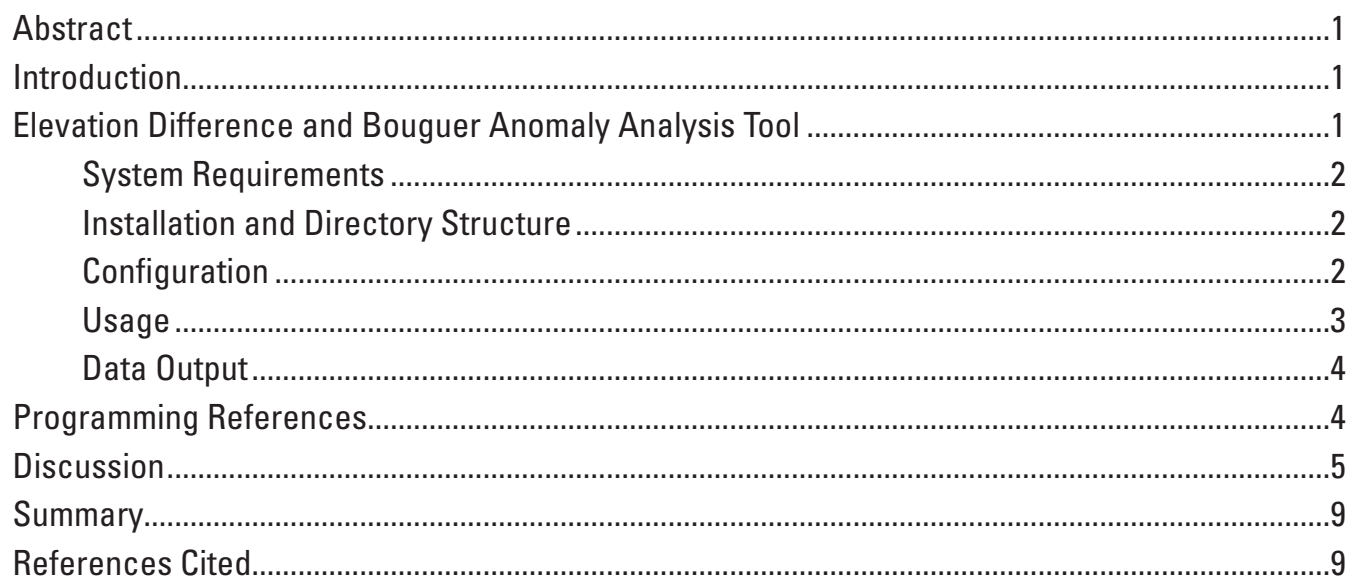

\section{Figures}

1. Screen capture showing "Configuration Manager" window of the Elevation Difference and Bouguer Anomaly Analysis Tool. This window allows the user to create or delete custom configuration and select which configuration will be used.

2. Screen capture showing "New Configuration" window of the Elevation Difference and Bouguer Anomaly Analysis Tool. This window allows the user to enter values for each configurable parameter and name the new configuration. Data type (float, int or String) and description (left-hand column) is shown for each parameter

3. Screen capture showing Elevation Difference and Bouguer Anomaly Analysis Tool configuration parameters

4. Screen capture showing latitude versus longitude plot of elevation difference shown by shape and color (no existing data provided). Example output from the Elevation Difference and Bouguer Anomaly Analysis Tool

5. Screen capture showing latitude versus longitude plot of elevation difference shown by color and shape (existing data provided). Example output from the Elevation Difference and Bouguer Anomaly Analysis Tool

6. Screen capture showing Bouguer Anomaly versus elevation plot. The solid black line represents the mean Bouguer anomaly at each elevation. The dotted black lines represent two standard deviations from the mean. Example output from the Elevation Difference and Bouguer Anomaly Analysis Tool

7. Screen capture showing Elevation Difference and Bouguer Anomaly Analysis Tool dialog window to select the part of data to be saved.....

\section{Tables}

1. Elevation Difference and Bouguer Anomaly Analysis Tool configuration parameters

2. Elevation Difference and Bouguer Anomaly Analysis Tool field descriptions 


\section{Conversion Factors}

\begin{tabular}{lccc}
\multicolumn{2}{l}{ International System of Units to U.S. customary units } & & \\
\hline & Multiply & By & To obtain \\
\hline & Length & \\
\hline meter $(\mathrm{m})$ & 3.281 & foot $(\mathrm{ft})$ & \\
\hline
\end{tabular}

\section{Abbreviations}

$\begin{array}{ll}\text { CSV } & \text { comma separated values } \\ \text { EDBAAT } & \text { Elevation Difference and Bouguer Anomaly Analysis Tool } \\ \text { GDUS } & \text { Gravity Database of the United States } \\ \text { JSON } & \text { JavaScript Object Notation } \\ \text { TNM } & \text { The National Map }\end{array}$




\title{
Elevation Difference and Bouguer Anomaly Analysis Tool (EDBAAT) User's Guide
}

\author{
By Aaron M. Smittle and Thomas G. Shoberg
}

\begin{abstract}
This report describes a software tool that imports gravity anomaly point data from the Gravity Database of the United States (GDUS) of the National Geospatial-Intelligence Agency and University of Texas at El Paso along with elevation data from The National Map (TNM) of the U.S. Geological Survey that lie within a user-specified geographic area of interest. Further, the tool integrates these two sets of data spatially and analyzes the consistency of the elevation of each gravity station from the GDUS with TNM elevation data; it also evaluates the consistency of gravity anomaly data within the GDUS data repository. The tool bins the GDUS data based on user-defined criteria of elevation misfit between the GDUS and TNM elevation data. It also provides users with a list of points from the GDUS data, which have Bouguer anomaly values that are considered outliers (two standard deviations or greater) with respect to other nearby GDUS anomaly data. "Nearby" can be defined by the user at time of execution. These outputs should allow users to quickly and efficiently choose which points from the GDUS would be most useful in reconnaissance studies or in augmenting and extending the range of individual gravity studies.
\end{abstract}

\section{Introduction}

The ability to augment local gravity surveys or make preliminary reconnaissance determinations using data from the World Wide Web can enhance areal coverage and spatial resolution of a preexisting gravity survey and reduce acquisition costs by eliminating redundant stations in a newly designed survey. The Gravity Database of the United States (GDUS) hosted by the University of Texas at El Paso and sponsored by the National Geospatial-Intelligence Agency is the largest such repository of gravity point data for the United States consisting of more than 1.2 million records (Keller and others, 2006). Although all the gravity data within this database were reduced in the same manner (Hinze and others, 2005), there is considerable variation and little documentation for the geospatial data associated with each gravity station. Recently, Shoberg and Stoddard (2013) developed a statistical method to seamlessly integrate GDUS gravity data with local global positioning system-controlled gravity stations and data layers from The National Map (TNM) of the U.S. Geological Survey. The tool described in this report, the Elevation Difference and Bouguer Anomaly Analysis Tool (EDBAAT), uses a part of this method to evaluate the consistency of GDUS station geospatial information with elevation data from TNM. This tool will enable users to identify a set of points from the GDUS for which confidence would be high in terms of elevation control and, as such, would be good candidates to augment local surveys or for planning purposes.

\section{Elevation Difference and Bouguer Anomaly Analysis Tool}

The EDBAAT extracts gravity station data from the GDUS and elevation data from TNM within a geographic bounding box defined by user-generated edge coordinates. This automated system then evaluates the misfit between the GDUS station elevation data and the elevation data from TNM. These are binned according to a user-defined system (for example, 0-3 meter [m] misfit, 3-5 m misfit, and greater than $5 \mathrm{~m}$ misfit); the lower misfit implies a more robust value for the GDUS gravity station elevation information. The tool also compares the gravity anomaly data for internal consistency. Any particular gravity value will be flagged as "questionable" if its value is more than two standard deviations from the mean, and at the same time is geographically near another gravity point that is within two standard deviations from the mean. The user defines what is geographically "nearby" at runtime. A report is then made available to the user listing gravity anomaly values of the outlier points (flagged as questionable) and those of the nearby points to aid in the decision on whether or not the user would like to keep or delete the flagged points from their data. Clearly, the implication is that large differences between outlier stations and those geographically near to them could indicate inconsistent gravity anomaly data in the outlier stations. Parallel computing is leveraged to increase speed of data retrieval. The steps required to use this tool are detailed below, beginning with system requirements and installation instructions. 


\section{System Requirements}

- Microsoft Windows version 7 or newer, Mac OS X version 10.8 or newer, or Linux operating system. A multicore system is preferred but not required.

- Python version 2.7 installed (https://www.python.org/ download/releases/2.7/). Version 2.7.5 or later recommended.

- The numpy Python package installed, which is available through the Python package manager "pip" or from the numpy releases page (https://github. com/numpy/numpy/releases). Version 1.7.1 or later required.

- The matplotlib Python package installed, which is available through the Python package manager, "pip," or by following instructions on the matplotlib Web site (http://matplotlib.org/users/installing.html). Version 1.3.0 or later required.

- Internet connection.

- At least 50 megabytes free on hard drive.

- At least 1 gigabyte of random access memory (RAM) recommended. The EDBAAT can work with less, but processing regions with thousands of points may be very slow.

\section{Installation and Directory Structure}

The EDBAAT package is written in Python and requires minimal installation efforts after retrieving the distribution package. The user starts by unpacking the zip file to a hard drive. This action will create a directory called "EDBAAT." This base directory contains all code and required files for EDBAAT to run properly. The base directory contains several subdirectories: "config," "out," "source," and "tests." The "config" directory contains the configuration file containing the default and any custom configurations; this directory should not be edited by the user at any point. The "out" directory exists as a suggested location for output files and contains several examples of output files in different possible formats. The "source" directory contains the Python code that is required to compile EDBAAT. The "tests" directory contains several test datasets that may be used to verify the tool is properly retrieving and parsing data from its sources.

In addition to these subdirectories, "EDBAAT" also contains three files of note: "_ init_.py," "test.py," and "EDBAAT.py." The "_ init_.py" file is required for this package to be a complete module. The file allows other Python programs to import this tool and use its capabilities. The "test.py" file can be launched to run all tests in the tests directory to verify proper data retrieval. Finally, "EDBAAT. py" will launch EDBAAT.

The EDBAAT tool can be downloaded from the website: https://cegis.usgs.gov/data_integration.html.

\section{Configuration}

The EDBAAT allows the user to specify particular query parameters or rely on a standard default setting. The parameters are changed using the "Configuration Manager" tool. Upon launching EDBAAT, the user is asked if they would like to update the configuration used by EDBAAT. Selecting yes will launch the EDBAAT "Configuration Manager" tool (fig. 1). From this tool, the user has the option to create a new configuration (fig. 2), copy an existing configuration, use the default value (table 1), or delete a custom configuration. Choosing an option to change the parameters will open a configuration window preloaded with previously entered values for each parameter (this is useful for creating a configuration that is similar to an existing configuration and modifying only some of the values). It is important to note that when copy-

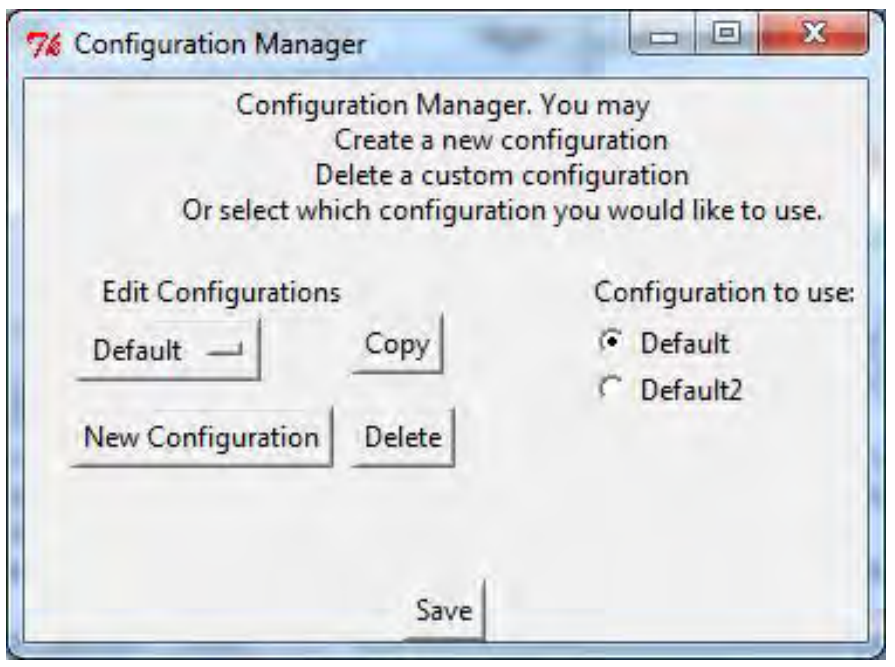

Figure 1. "Configuration Manager" window of the Elevation Difference and Bouguer Anomaly Analysis Tool. This window allows the user to create or delete custom configuration and select which configuration will be used.

ing and deleting a configuration, selection is made using the drop-down menu in the top left under "Edit Configurations" and not by the radio buttons on the right. The radio buttons on the right side (under "Configuration to use") are used to select the configuration EDBAAT will use when it is run. The parameters necessary for tool configuration are cataloged in table 1 .

It is also important to note that the user may not edit or delete the "Default" configuration. An attempt to create a new configuration with the name "Default" will force the user to rename the new configuration file. The EDBAAT "Configuration Manager" tool does not, however, prevent the user from 


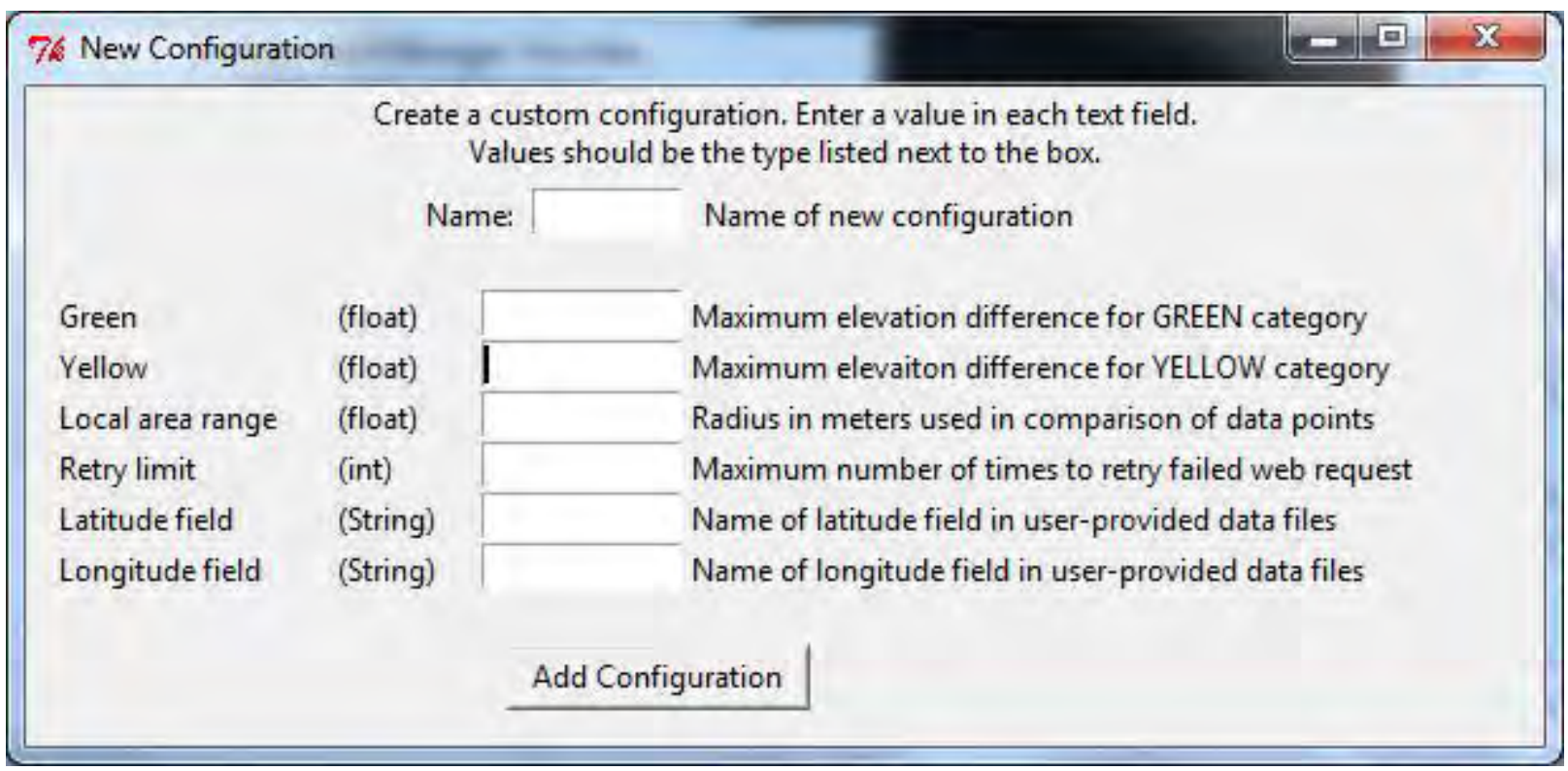

Figure 2. "New Configuration" window of the Elevation Difference and Bouguer Anomaly Analysis Tool. This window allows the user to enter values for each configurable parameter and name the new configuration. Data type (float, int or String) and description (left-hand column) is shown for each parameter.

Table 1. Elevation difference and Bouguer Anomaly Analysis Tool configuration parameters.

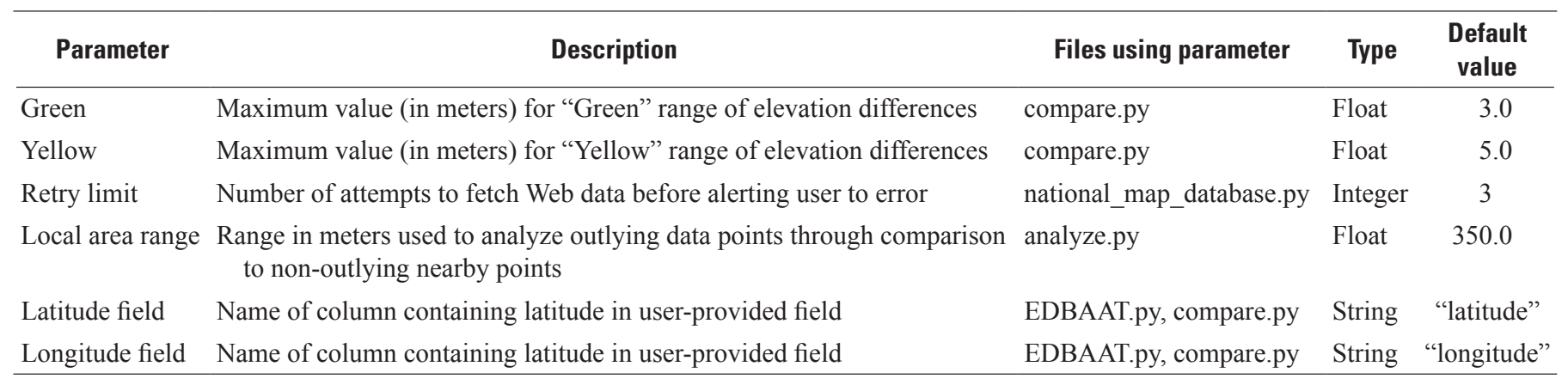

creating a new configuration with any variation of "Default" as the name (including altering whitespace, capitalization, or spelling).

\section{Usage}

The EDBAAT may be launched by double clicking the "EDBAAT.py" script in the "EDBAAT" directory (Microsoft Windows and Linux), double clicking the EDBAAT application icon (Mac OS X), or from a command line environment (Command Prompt on Microsoft Windows, Terminal on Mac OS X, or various programs based on Linux distribution). Generally, in a Microsoft Windows and Linux operating system, double clicking a Python script will execute that script; however, in a Mac OS X operating system, double clicking on a Python script typically opens the script in an editor. For simplified running, a wrapper has been included to allow Mac OS X users to double click an application file to run the tool. To run EDBAAT from a command line, navigate to the base directory using "cd" (change directory). Once in the "EDBAAT" directory, EDBAAT can be launched with this command:

python EDBAAT.py

When EDBAAT is launched, the main window containing input boxes for the geographic bounding box of the tool are displayed (fig. 3). Enter the latitude and longitude (in decimal degrees) into the boxes to run the tool. The tool retrieves all data from the two sources and compares the reported 


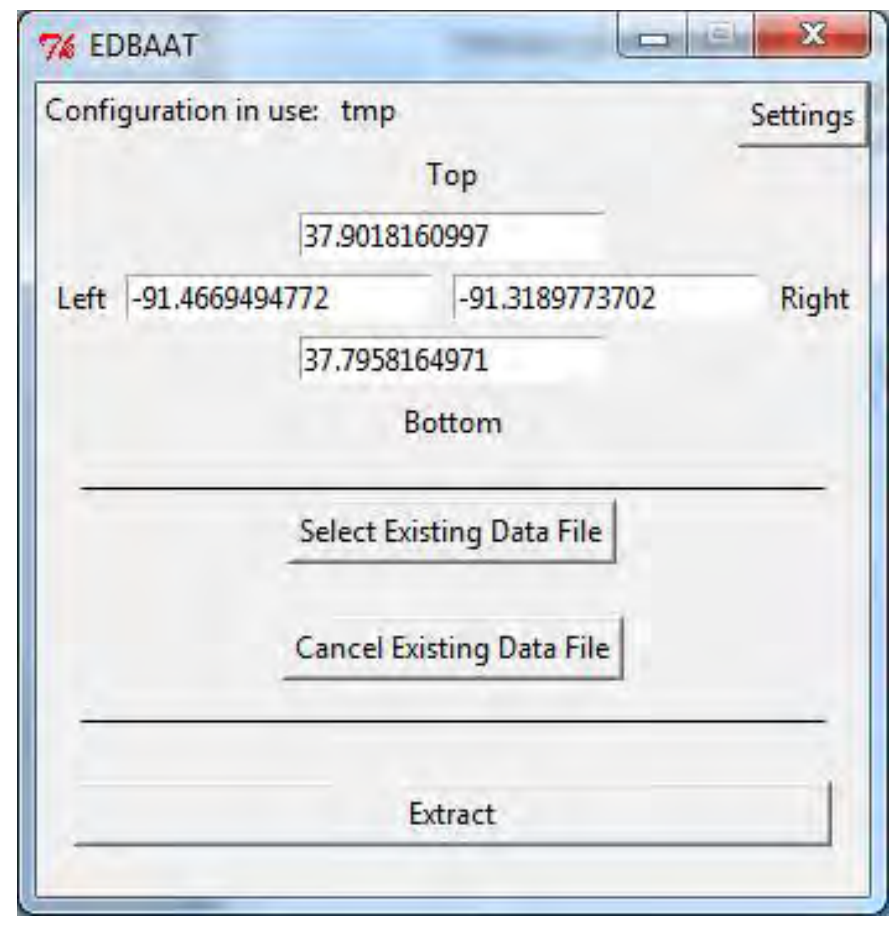

Figure 3. Elevation Difference and Bouguer Anomaly Analysis Tool configuration parameters.

elevation. Each data point is then plotted by latitude and longitude, with color and shape denoting a range of elevation differences specified in the configuration (fig. 4). If an existing data file was provided, the points from that file will also be displayed on the plot (fig. 5).

At this point, the EDBAAT may be returned to the main window to select a different search area, or the user can request further analysis of the Bouguer anomalies. A statistical analysis of Bouguer anomalies can be obtained by graphing the Bouguer anomalies versus elevation (fig. 6). In this plot, a blue dot represents any point within two standard deviations of the mean. A yellow diamond represents a point outside that range. A red hexagon represents a point outside two standard deviations from the mean but in close geographic proximity (specified through the configuration as "Local area range") to another gravity station. After running the analysis of the Bouguer anomalies, the data may be saved in one of two formats: a JavaScript Object Notation (JSON) or comma separated values (CSV) file. Further, the data to be saved can be selected to only include points in a given category (nonoutlier data by selecting "Non-outlying points," outlier data by selecting "Outlying points," or data that have been flagged as questionable by internal consistency by selecting "Possibly in Error") or by latitude and longitude using another bounding box (fig. 7). Upon saving these sorted data, a file may be created linking each data point flagged as questionable to the stations that fall within the proscribed proximity of these questionable stations.

\section{Data Output}

Data from the EDBAAT tool may be saved as either a JSON or CSV file. In a CSV file, field names are modified to reflect the field and source of the data. This is because CSV is an inherently flat data format (each column can only contain a value, rather than a set of columns and values). In order to show the source of each field, field names are prefixed with "grav data " for fields coming from the GDUS and "nat map_data_" for fields coming from TNM. Additionally, CSV files contain a flag field used to denote if that data point is nonoutlying, outlying, or flagged as possibly in error. In JSON files, the data are stored in a single object with a field for each category. Each field corresponds to a list of all data in that category. Because JSON files allow for nesting objects, there is no modification to any field names in the data.

The data saved to link data points flagged as questionable with their nearby data points that fall within a given proximity are stored as a simple text file. This file contains a series of arrays where the first item is the point flagged as a possible error. The second item contains a list of all nearby points that are within the confidence limit.

The "source" directory contains a file, "file_utilities.py," which contains functions to parse an EDBAAT data file and return the data as a tuple in the form (nonoutlying, outlying, possibly in error). These functions are "jsonToTuple(file)" and "csvToTuple(file)." Each function takes a file object, opened in read mode, and parses the data.

A complete description of each field in the data, along with source and field name in each file format, is provided in table 2.

\section{Programming References}

The EDBAAT code was written to be simple and easy to understand by reading the documentation and code together. For the sake of completeness, a description of each file and its functions are provided here. Users unfamiliar with Python may want to refer to Python online tutorials and documentation (https://docs.python.org/2/).

The EDBAAT tool adheres to Python's PEP8 style guide in most cases (https://www.python.org/dev/peps/pep-0008/). The standard of lines not exceeding 80 characters has been abandoned to avoid multiline statements where readability may suffer.

Naming conventions and patterns are as follows (these may not strictly adhere to PEP8 standards where a deviation from the standard enhances readability): 


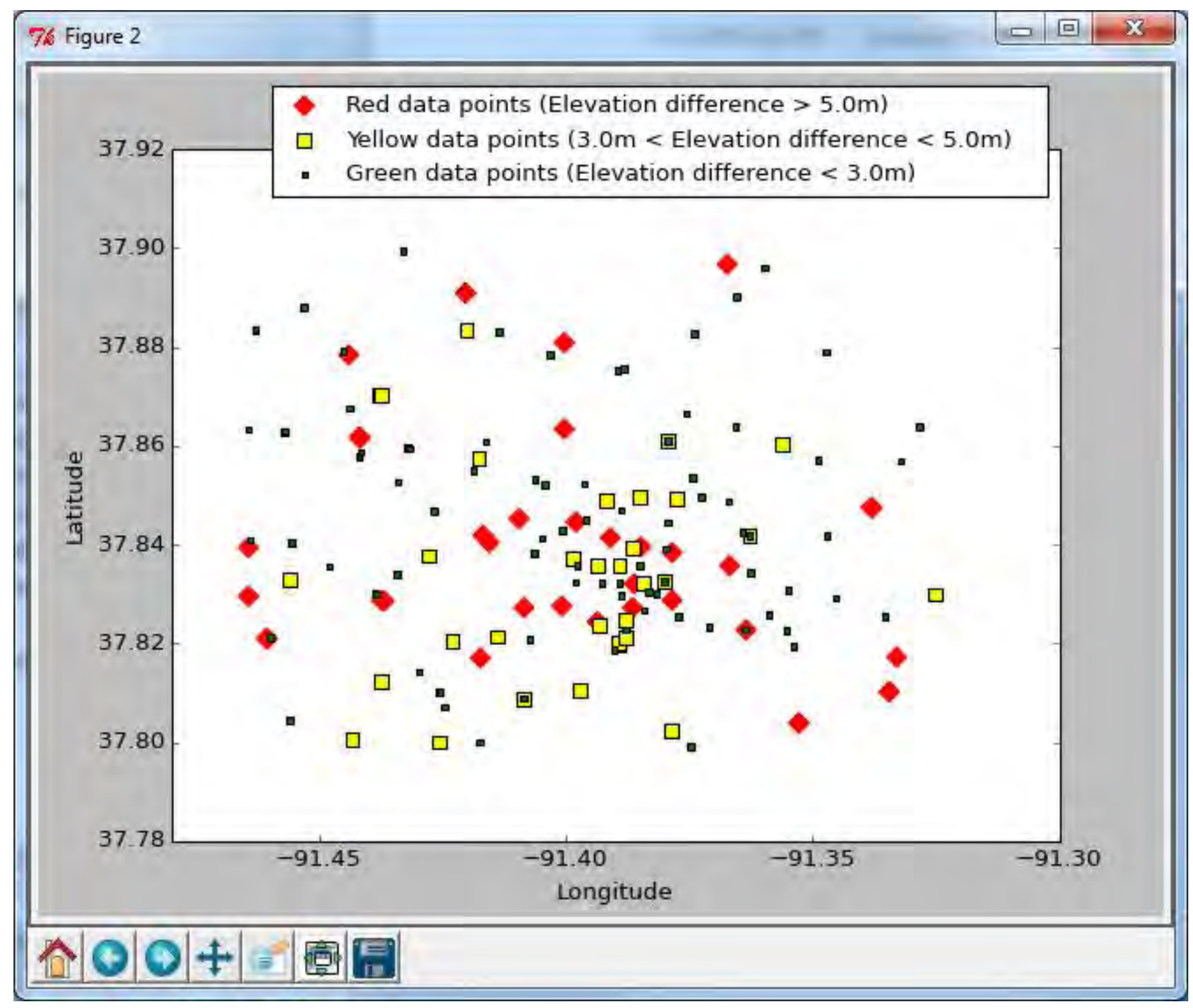

Figure 4. Latitude versus longitude plot of elevation difference shown by shape and color (no existing data provided). Example output from the Elevation Difference and Bouguer Anomaly Analysis Tool.

- Files are named using lowercase with underscores (for example, "nat_map_data"). Exceptions are the drivers for the complete tool ("EDBAAT.py" and "source/EDBAAT.py"), which are named in all capitals (for example, "EDBAAT"), and the configuration manager ("source/Configure.py"), which is named using capitalized words (also called "CamelCase;" for example, "ConfigureMapData") to show they are meant to run by the user (or, as is the case with "Configure.py," that they are fully functional and independent of the rest of the tool).

- Classes are named using capitalized words.

- Class member variables adhere to the same naming conventions as other variables with the exception of Buttons in graphical user interface classes. Buttons are named in all capitals to make it easy to distinguish a button name from a label or local variable.

- Local variables are named using lowercase with underscores $\left(\_\right.$). Variable names containing a number separate the number from words with underscores (for example, "var_1" instead of "var1").

Every class and function has a descriptive docstring. A class docstring contains a high-level description of the class, whereas a function docstring contains a description of the function's behavior, an explanation of each parameter (including expected type), and the return value (and type). 
Table 2. Elevation difference and Bouguer Anomaly Analysis Tool field descriptions.

\begin{tabular}{|c|c|c|c|}
\hline Data source & Field name in .json file & Field name in .csv file & Field description \\
\hline Gravity data & cbanom 267 & grav_data_cbanom 267 & $\begin{array}{l}\text { Complete Bouguer anomaly reduced to a back- } \\
\text { ground density of } 267\end{array}$ \\
\hline Gravity data & elevngvd $88 \mathrm{~m}$ & grav_data_elevngvd $88 \mathrm{~m}$ & $\begin{array}{l}\text { Elevation using North American Vertical Datum of } \\
1988 \text { (meters) }\end{array}$ \\
\hline Gravity data & itc & grav_data_itc & Inner terrain correction \\
\hline Gravity data & latnad27 & grav_data_latnad27 & Latitude using North American Datum of 1927 \\
\hline Gravity data & latnad83 & grav_data_latnad83 & Latitude using North American Datum of 1983 \\
\hline Gravity data & lonnad 27 & grav_data_lonnad27 & Longitude using North American Datum of 1927 \\
\hline Gravity data & sourcecode & grav_data_sourcecode & Reference to who collected data or assigned ID \\
\hline The National Map & Data_Source & nat_map_data_Data_Source & The source survey or study for the elevation data ${ }^{1}$ \\
\hline The National Map & $\mathrm{x}$ & nat_map_data_x & Longitude \\
\hline The National Map & $\mathrm{y}$ & nat_map_data_y & Latitude \\
\hline The National Map & Elevation & nat_map_data_Elevation & Elevation of point \\
\hline The National Map & Units & nat_map_data_Units & Unit of measurement for elevation \\
\hline [Generated] & difference & difference & Difference in elevation between two sources \\
\hline [Generated] & category & category & Color-coded category based on elevation difference \\
\hline [Generated] & $\begin{array}{l}\text { [Does not exist, is specified in } \\
\text { file through labels] }\end{array}$ & flag & $\begin{array}{l}\text { Flag value to differentiate data types (outlier or } \\
\text { probably in error, blank if non-outlier) }\end{array}$ \\
\hline
\end{tabular}

${ }^{1}$ As of December 7, 2015, all data comes from 3D Elevation Program 1/3 arc-second.

\section{Discussion}

The EDBAAT is described in this report. The EDBAAT imports all gravity data from the GDUS that lie within a user-specified geographic bounding box. These data are then evaluated for reliability based on two criteria. The first checks the geospatial data for the GDUS against that of TNM; in particular, the elevation data are critiqued because correct gravity anomaly interpretation is most sensitive to the elevation accuracy of a station location. Stations are binned based on the difference in elevation between the station's geospatial data and that of the same latitude and longitude as derived from TMN's digital elevation model. The user is left to choose how much elevation difference is acceptable for including any given gravity anomaly point into a survey. The second check for data point reliability considers the average regional trend of gravity in the user-specified geographic bounding box. Here the anomaly data are plotted versus elevation and a regional trend (regression line) is determined, along with its 95 -percent confidence limits. The gravity stations are then flagged based on whether or not they are outlying or inlying of these confidence limits, and whether or not they constitute a questionable gravity reading (lying outside the 95-percent confidence limit, and yet at the same time lying geographically close [the default value of close is within 350 meters, but this can be controlled by the user] to another station that is not outside the 95-percent confidence limit). This flag does not tell the user which, if either, point is least reliable but does portray points that should be looked at more closely before including them into a final analysis.

The major benefits of the EDBAAT is (1) to enable a user to extract a set of gravity stations within an area of interest from a previous study and (2) calculate a statistically based measure of accuracy for the geospatial and geophysical data. In turn, these confidence measures are useful to scientists examining the data. The tool does allow a preexisting survey or a set of longitude and latitude coordinates for a proposed survey to be entered and a scatter plot created. This can inform investigators about the usability of the additional stations from the GDUS for preliminary reconnaissance of an area in which a gravity survey is warranted, or to augment a survey with additional data, or, to increase the areal extent of a survey, or any combination of these options. 


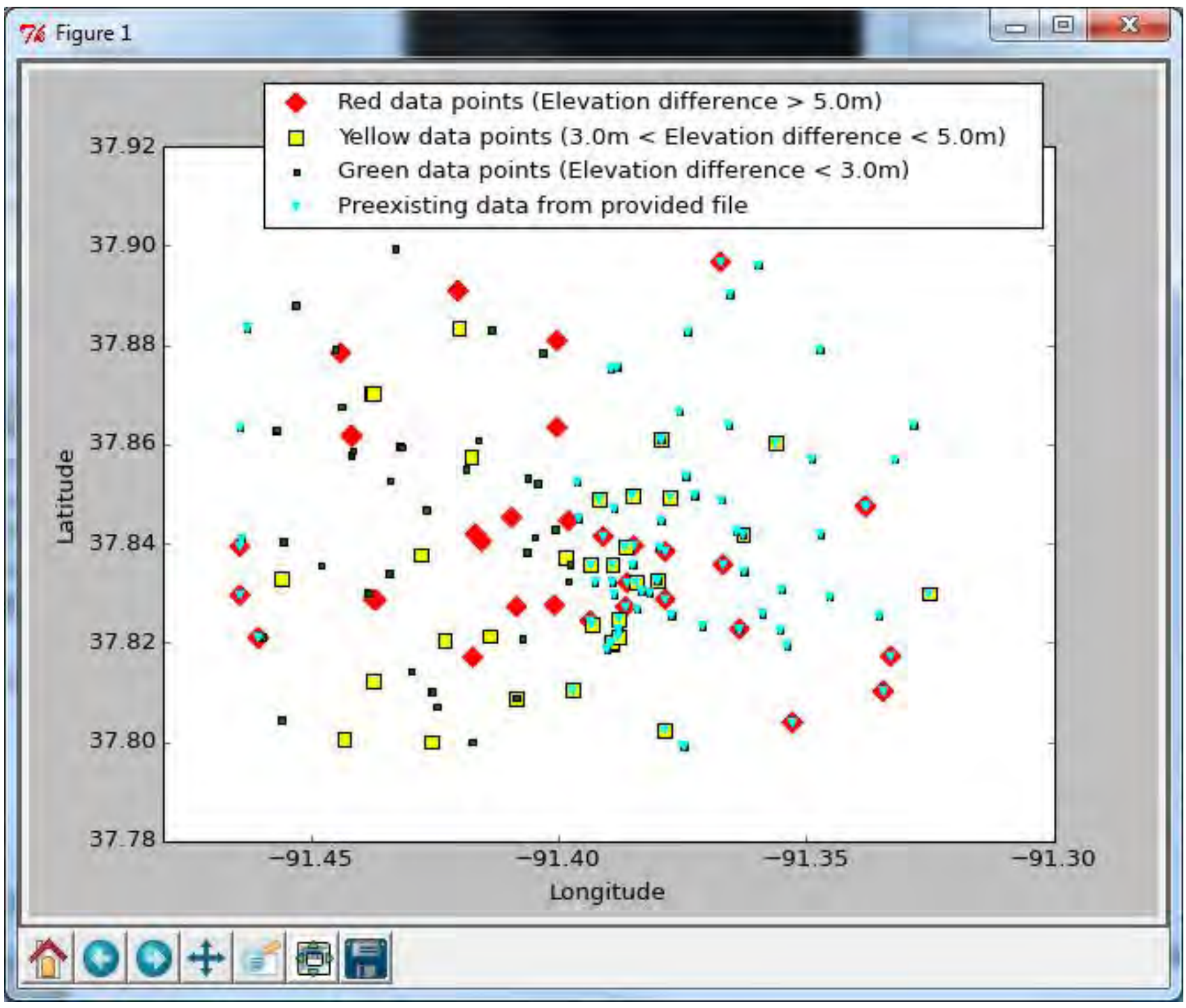

Figure 5. Latitude versus longitude plot of elevation difference shown by color and shape (existing data provided). Example output from the Elevation Difference and Bouguer Anomaly Analysis Tool. 


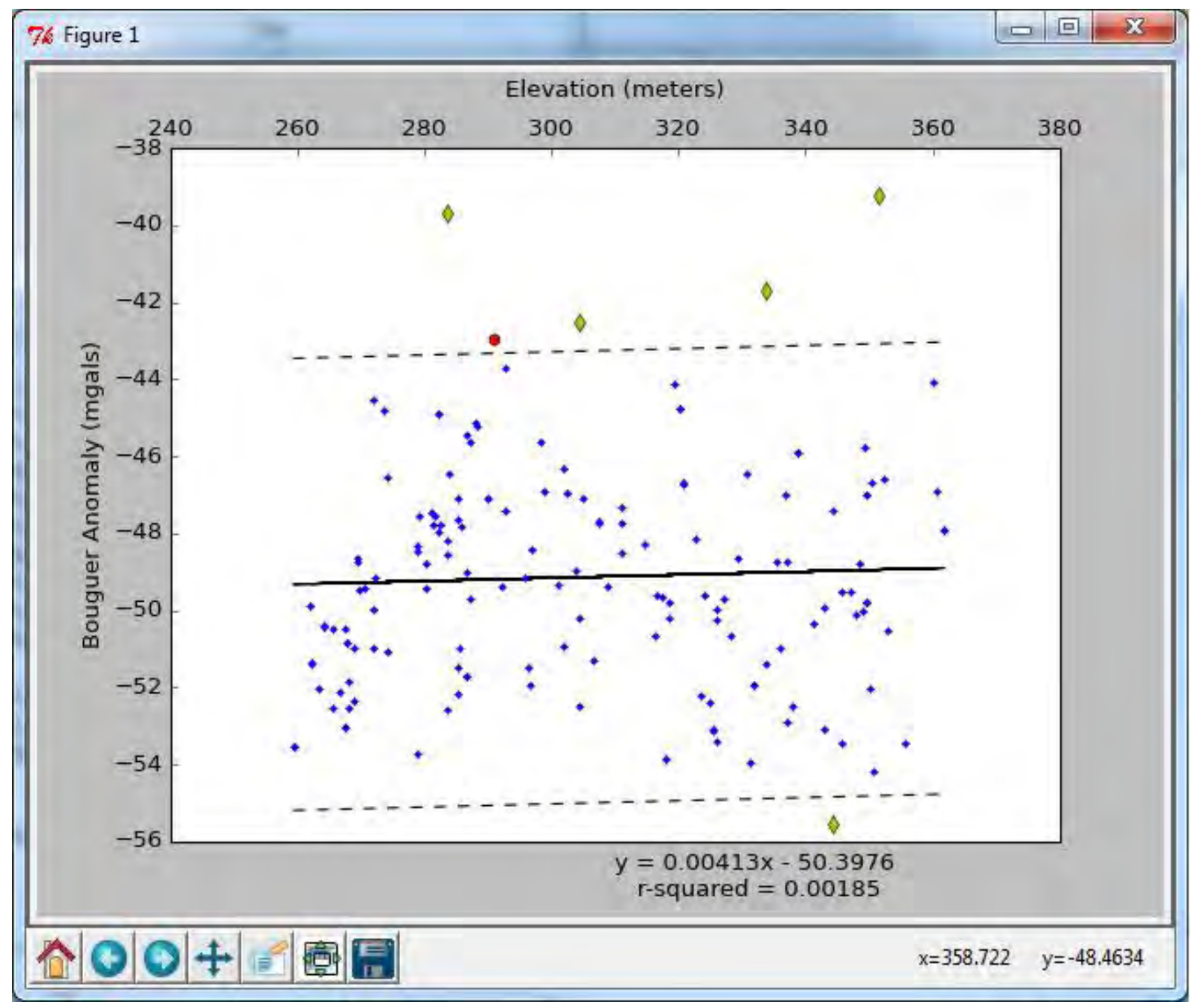

Figure 6. Bouguer Anomaly versus elevation plot. The solid black line represents the mean Bouguer anomaly at each elevation. The dotted black lines represent two standard deviations from the mean. Example output from the Elevation Difference and Bouguer Anomaly Analysis Tool. 


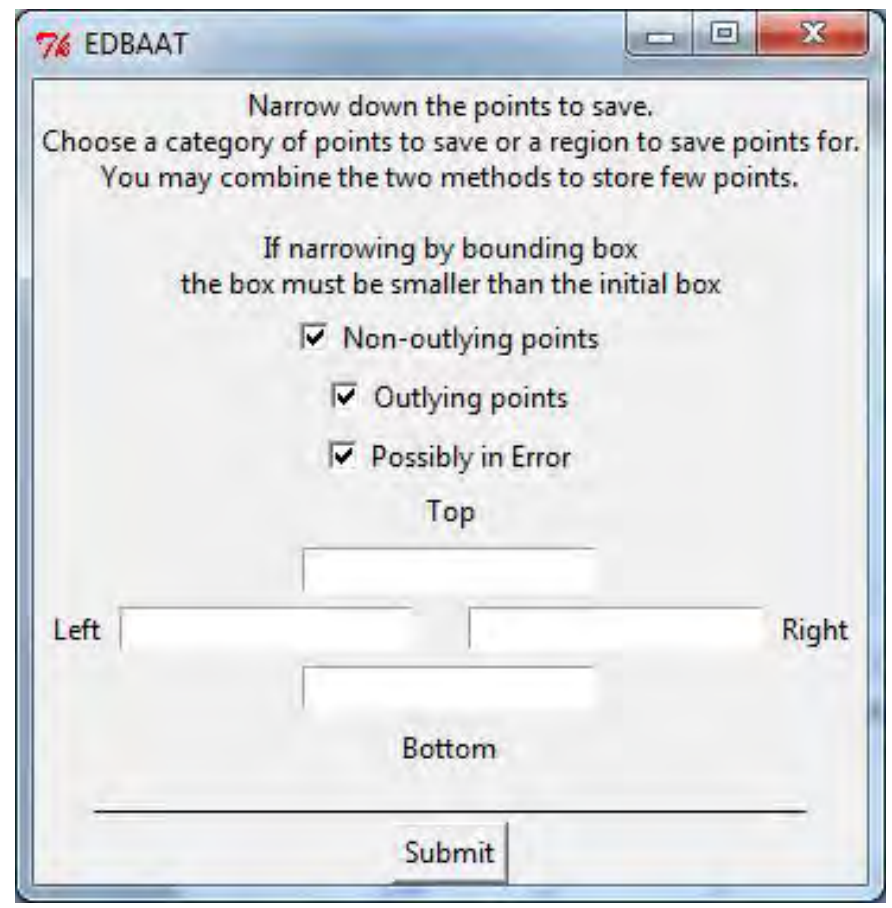

Figure7. Elevation Difference and Bouguer Anomaly Analysis Tool dialog window to select the part of data to be saved.

\section{Summary}

The web-based Elevation Difference and Bouguer Anomaly Analysis Tool (EDBAAT) software tool imports gravity anomaly point data from the Gravity Database of the United States (GDUS) of the National Geospatial-Intelligence Agency and University of Texas at El Paso along with elevation data from The National Map (TNM) of the U.S. Geological Survey that lie within a user-specified geographic area of interest. The tool then spatially integrates these data and analyzes the consistency of the elevation for each gravity station; it also evaluates the consistency of gravity anomaly values for each station within the GDUS data repository. It provides users with a list of points from the GDUS data, which have Bouguer anomaly values that are considered outlying (two standard deviations or greater) with respect to other nearby gravity data. "Nearby" can be defined by the user at time of execution. The tool also bins the GDUS data based on user-defined criteria of elevation misfit between the GDUS and TNM elevation data. All of this information can be output to allow the user to quickly and efficiently choose which points from the GDUS would be most useful in reconnaissance studies or in augmenting and extending the range of individual gravity studies.

\section{References Cited}

Hinze, W.J.; Aiken, Carlos; Borzena, John; Coakley, Bernard; Dater, David; Flanagan, Guy; Forsberg, René; Hildenbrand, Thomas; Keller, G.R.; Kellogg, James; Kucks, Robert; Li, Xiong; Mainville, Andre; Morin, Robert; Pilkington, Mark; Plouff, Donald; Ravat, Dhananjoy; Roman, Daniel; UrrutiaFucugauchi, Jamie; Véronneau, Marc; Webring, Michael; Winester, Daniel, 2005. New standards for reducing gravity data-The North American gravity database: Geophysics, v. 70, no. 4, p. J25-J32. [Also available at https://doi. org/10.1190/1.1988183.]

Keller, G.R.; Hildenbrand, T.G.; Kucks, Robert; Webring, Michael; Briesacher, Allen; Rujawitz, Kristine; Hittleman, A.M.; Roman, D.R.; Winester, Daniel; Aldouri, R.; Seeley, John; Rasillo, Jorge; Torres, Roberto; Hinze, W.J.; Gates, Ann; Kreinovich, Vladik; and Salayandia, Leonardo, 2006, A community effort to construct a gravity database for the United States and an associated Web portal: GSA Special Papers, v. 397, p. 21-34. [Also available at https://doi. org/10.1130/2006.2397(02).]

Shoberg, T.G., and Stoddard, P.R., 2013, Integrating stations from the North America Gravity Database into a local GPSbased land gravity survey: Journal of Applied Geophysics, v. 89, p. 76-83. [Also available at https://doi.org/10.1016/j. jappgeo.2012.11.011.] 
Publishing support provided by:

Rolla Publishing Service Center

For additional information concerning this publication, contact: Director, USGS National Geospatial Technical

Operations Center (NGTOC)

1400 Independence Road

Rolla, M0 65401

(573) 308-3500

Or visit the NGTOC website at: https://ngtoc.usgs.gov 


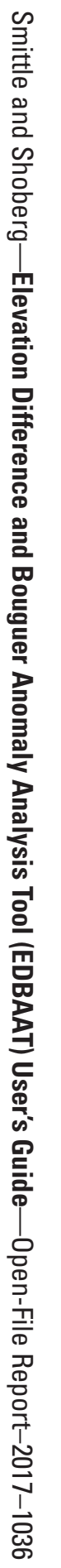

